\title{
Ion and electron ghost imaging
}

\author{
A. Trimeche, ${ }^{*}$ C. Lopez, ${ }^{\dagger}$ D. Comparat $\odot$, and Y. J. Picard ${ }^{\ddagger}$ \\ Université Paris-Saclay, CNRS, Laboratoire Aimé Cotton, 91405 Orsay, France.
}

(Received 4 March 2020; revised 29 June 2020; accepted 10 November 2020; published 30 November 2020)

\begin{abstract}
In this paper, we report a demonstration of ion and electron ghost imaging. Two beams of correlated ions and electrons are produced by a photoionization process and accelerated into opposite directions. Using a single time- and position-sensitive detector for one beam, we can image an object seen by the other beam even when the detector that sees this object has no spatial resolution. The extra information given by this second detector can, therefore, be used to reconstruct the image thanks to the correlation between the ions and the electrons. In our example, a metallic mask placed in front of a time-sensitive detector is used as the object to image. We demonstrate ion and electron ghost imaging using this mask in a transmission mode. These primary results are very promising and open applications especially in ion and electron imaging in surface science and nanophysics.
\end{abstract}

DOI: 10.1103/PhysRevResearch.2.043295

\section{INTRODUCTION}

Ghost imaging, also called "coincidence imaging" or "correlated imaging," is able to produce an image of an object even when the detector that sees the object has no spatial resolution. The requirement for this imaging is that a second detector collects a partner particle correlated to the one sent to the object. The extra information given by the second detector can therefore be used to infer the location of the first particle and can thus be used to reconstruct the image. Obviously, this method is very useful when spatially resolved detectors are unavailable or when the experimental architecture makes it difficult to implement one. It has been demonstrated using (entangled or correlated) photons [1-4], atoms [5], and with one photon and one electron (but, here, photons just serve as a source for the electrons created using a photocathode) [6].

Here we present this method using correlation between one electron and one ion. We demonstrate both ghost ion imaging using a spatially resolved electron detector and ghost electron imaging using a spatially resolved ion detector. These methods can then be applied to electron- or ion-based imaging systems to reduce the image acquisition time or to reduce the sample damage by reducing the amount of particles sent to the sample [7]. This can be combined with compressed sensing,

\footnotetext{
*Present address: Université Paris-Saclay, Institut d'Optique Graduate School, CNRS, Laboratoire Charles Fabry, 91127 Palaiseau, France.

†Present address: Université Paris-Saclay, ENS Paris-Saclay, LUMIN, 91405 Orsay, France.

${ }^{\ddagger}$ Corresponding author: yan.picard@ universite-paris-saclay.fr

Published by the American Physical Society under the terms of the Creative Commons Attribution 4.0 International license. Further distribution of this work must maintain attribution to the author(s) and the published article's title, journal citation, and DOI.
}

optimizing the sparsity of a signal [8], and can thus improve the contrast compared to a conventional image [9]. Indeed, when the reference intensity pattern that is sent to a sample is perfectly known, it opens the way to clever algorithms such as compressed or denoising imaging ones, for instance, for a sample with a sparse image [10]. As an illustration, in our case, because of the corresponding particle detection, the sent particle number and location are perfectly known (in a sub-Poissonian deterministic way), whereas in a standard, non-ghost-imaging thermal source, the underlying intensity is (fractional) Poissonian and shot-noise limited. To give an extreme, but illustrative ideal example, in standard imaging techniques, due to the unknown number of particles hitting a sample (Poisson distribution), one has to send at least a few electrons to see the contrast between a low and a high secondary electron yield sample. In contrast, in a ghost imaging, because of the detection of the correlated (ion ghost) particle, we know the exact number of electrons hitting the sample and, therefore, only one electron is enough to see whether or not it produces secondary electrons.

Furthermore, our method will open some spatial resolution capabilities to spectroscopic methods having usually poor spatial resolutions [11] [high-resolution electron energy-loss spectroscopy (HREELS) being one obvious example].

\section{EXPERIMENTAL APPARATUS}

The experimental setup, designed to produce beams of correlated ions and electrons, has been described in detail previously [12]. The aim of this previous work was to improve a deterministic single-ion source by controlling the position and momentum of each ion. Based on the extra information given by the electron created in a photoionization process, the initial position of the correlated ion was inferred and thus its trajectory was controlled using a real-time feedback system. The same experimental setup is used for the present work, except that only one of the correlated particles (ion or 
electron) created in a photoionization process will be detected with a position-sensitive detector and, for the other one, the arrival position will not be measured.

The setup is based on a double time-of-flight (TOF) spectrometer with detectors at opposite ends monitored in coincidence mode. For this experiment, we use cesium (Cs) atoms so as to create ion-electron pairs. Cs effuses from an oven and propagates to the ionization region. Near-threshold photoionization is performed in a static electric field produced between two holed electrodes. By using narrowband lasers for the photoionization, Doppler selection is performed to drastically reduce the effect of the effusive atomic beam velocity dispersion. Ionization of Cs atoms is performed with a three-photon transition process, where the first transition uses a horizontal laser beam counterpropagating with respect to the atomic beam. It is ensured by a $852 \mathrm{~nm}$ laser beam that excites atoms from the $6 S_{1 / 2} F=4$ level to the $6 P_{3 / 2}$ $F=5$ level. For Doppler compensation, this laser is locked by saturated absorption on a Cs vapor cell and detuned by an acoustic optical modulator. The second transition is performed by a perpendicular $1470 \mathrm{~nm}$ laser beam coupling the excited $6 P_{3 / 2}$ state to the $7 S_{1 / 2}$ state. This laser is locked thanks to the two-photon process excitation of a Cs cell excited by the $852 \mathrm{~nm}$ laser. The last transition is ensured by a tunable Ti:sapphire laser, in the same plane but tilted $45^{\circ}$ from the previous lasers. It excites the atoms from the $7 S_{1 / 2}$ state to a Rydberg state or the ionization continuum, depending on the chosen wavelength $(770-795 \mathrm{~nm})$. This geometry enables us to control the ionization volume at the crossing of the laser beams. These lasers are focused, inside the extraction zone, with a typical size of $\simeq 500 \mu \mathrm{m}$. For the results presented here, ionization occurs in a $2200 \mathrm{~V} / \mathrm{cm}$ field and the (vacuum) Ti:sapphire laser wavelength is tuned to $794.432 \mathrm{~nm}$. Fine adjustments are made to the Ti:sapphire laser wavelength to optimize the detected electron and ion signals. We choose this wavelength because it results in an efficient excitation towards a Rydberg state that autoionizes, and produces an efficient source of ion and electron pairs. After ionization, the electron and ion are accelerated by the $2200 \mathrm{~V} / \mathrm{cm}$ static electric field in opposite directions toward the detectors. The time signals coming from each detector are digitized by constant fraction discriminators coupled to a multichannel time-to-digital converter. Typical TOF values, from the ionization region to the detectors, are about $20 \mathrm{~ns}$ for the electrons and $7 \mu \mathrm{s}$ for the ions. Since we use the same setup to obtain both ion and electron ghost images and for clarity purposes, the two correlated particles produced by the ionization will be called particles $A$ and $B$ in the next section, with $A$ being the one that sees the object to image and $B$ the one that will reveal the ghost image of the object.

\section{CORRELATION}

The correlation between an electron and ion allows one to infer the position of a particle from its correlated one. On one side of the spectrometer, the mask shown in Figs. 1(b) and 2(b) is placed in front of a time-sensitive detector (TSD) composed of a set of microchannel plates (MCPs) and a conductive anode. Thus, a particle $A$ hits this detector only if it passes
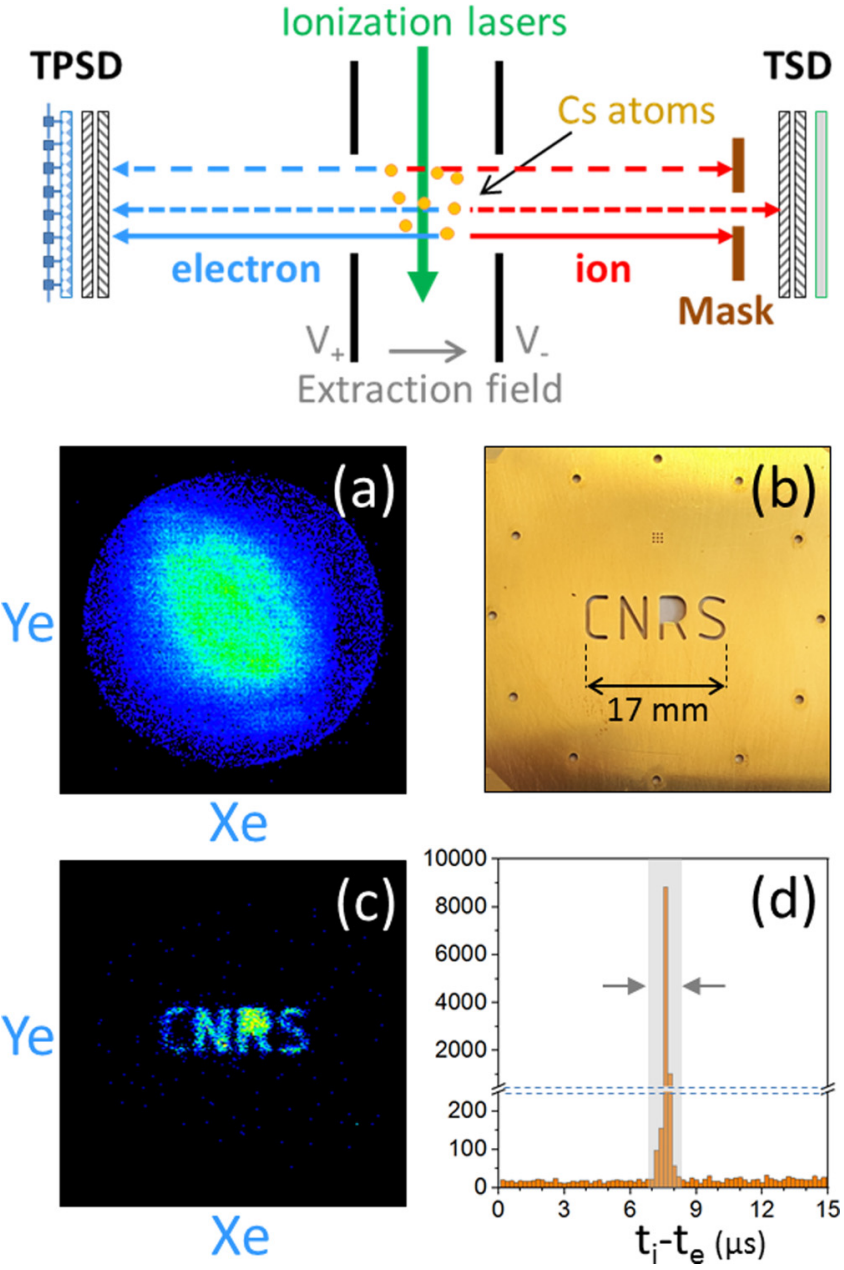

FIG. 1. Upper part: Scheme of the experimental setup in the ion ghost-imaging configuration. The ion-electron pair trajectories shown here are schematics and not to scale. (a) Image of the timeand position-sensitive detector (TPSD) for all the electrons (in blue). (b) Picture of the mask placed in front of the time-sensitive detector (TSD). (c) Image of the TPSD only for electrons that are detected in coincidence with their correlated ion. (d) Relative time-of-flight (TOF) histogram. The selection of the event whose relative TOF $\left(t_{i}-t_{e}\right)$ fits into the coincidence window (gray zone in between arrows) gives image (c), whereas the selection of all the events independently of their relative TOF gives image (a).

through the mask. Note that the front face of the TSD and the mask are maintained at the same voltage. The TOF of particle $A$ is measured, but its location on the detector cannot be determined since no device is set to measure the particle position on that side of the spectrometer. On the other side of the spectrometer, a time- and position-sensitive detector (TPSD) composed of a set of $40 \mathrm{~mm}$ open diameter MCPs and a delay line device measure the TOF and the position of the correlated particle $B$. This detector has a position resolution of about $50 \mu \mathrm{m}$. The TOF of particle $A$ and the TOF and position coordinates $X, Y$ of particle $B$ are handled by a $\mathrm{C}++$ monitoring home-written program that controls acquisition and stores the data. For each detected event, the acquisition program determines the position of particle $B$ and relative TOF of particles $A$ and $B$ with a $100 \mathrm{ps}$ resolution. If this relative TOF 

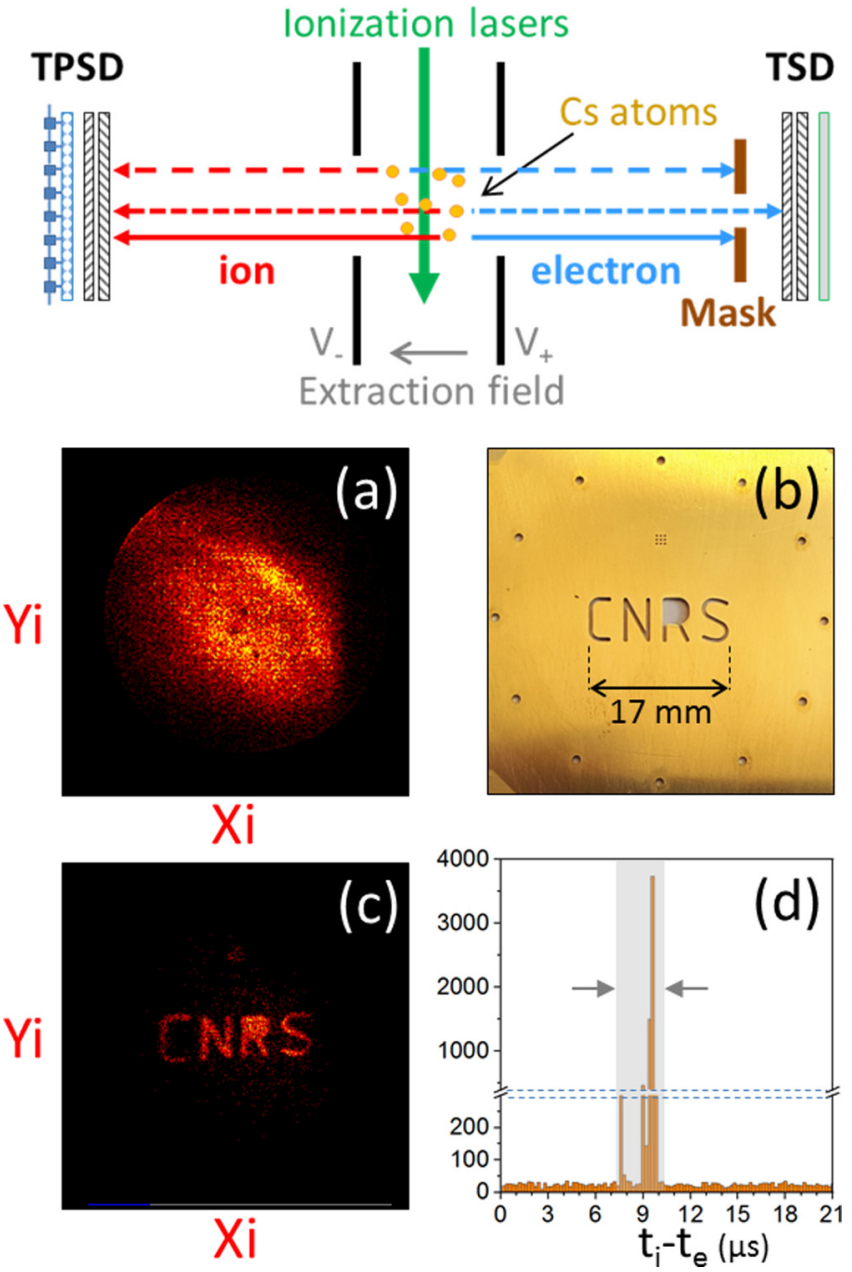

FIG. 2. Upper part: Scheme of the experimental setup in the electron ghost-imaging configuration. The ion-electron pair trajectories shown here are schematics and not to scale. (a) Image of the time- and position-sensitive detector (TPSD) for all the ions (in red). (b) Picture of the mask placed in front of the time-sensitive detector (TSD). (c) Image of the TPSD only for ions that are detected in coincidence with their correlated electron. (d) Relative time-of-flight (TOF) histogram. The selection of the event whose relative TOF $\left(t_{i}-t_{e}\right)$ fits into the coincidence window (gray zone in between arrows) gives image (c), whereas the selection of all the events independently of their relative TOF gives image (a).

fits in a given range ( $\simeq 200 \mathrm{~ns}$, corresponding to the spread caused by the potential difference across the ionization region) around the expected value, the pair is considered as coming from a unique ionization event and is labeled coincident in time.

The image obtained from the TPSD can thus be displayed only for the particles $B$ that are measured in coincidence with their correlated particle $A$ to reconstruct the so-called ghost image.

\section{ION GHOST IMAGING}

We first start with the configuration called ion ghost imaging (iGI), where all the voltages are set in a way that the electrons are accelerated toward the TPSD and the ions are accelerated toward the mask and the TSD, as shown in the scheme of Fig. 1. The mask used in this proof-of-principle experiment [Fig. 1(b)] is an 80-mm-diameter brass disk in which we engraved the $0.3 \mathrm{~mm}$ linewidth "CNRS" characters and some holes made for centering purpose. In this case, the ions (in red) will only be detected if they pass through the letters of the mask. On the other side, the extracted electrons (in blue) fly through the opposite part of the spectrometer and are all detected by the TPSD. Figure 1(a) shows the raw image of all the electrons detected by the TPSD at the end of an acquisition sequence. Without any treatment, this image can only inform us about the beam spread and its initial conditions. However, if we display only the electrons that are each in coincidence with an ion using the TOF signals delivered by the TSD and the TPSD, we obtain the image displayed in Fig. 1(c). Here we clearly see on this selected TPSD image a ghost image of the mask placed in front of the TSD. This imaging process is based on the coincidence criterion and the position correlation quality.

The coincidence criterion ensures that the detected electron and ion pairs are each coming from the same ionization event. This is validated by the measurement of the relative TOF of the two charged particles. The electron-ion pairs are created and accelerated in two opposite directions, and thus their relative TOF depends mainly on the acceleration voltage and the distances between the extraction zone and the correspondent detectors. As seen in Fig. 1(d), the electron-ion relative TOF in this iGI configuration is about 7-8 $\mu \mathrm{s}$. All the events that are outside this relative TOF window correspond to false coincidence, which means signals that are not coming from a $\mathrm{Cs}$ ionization event, such as noise, dark counts, missing counts, or a different ionized element than Cs.

The position correlation quality ensure that for the detected ions that pass through the mask, their corresponding coincident electrons will have a specific spatial distribution. The quality of link between the ion and electron positions depends on the accuracy of the correlation between them. If there is no correlation between the coincident charged particles, the image obtained after filtering the coincident electrons would also be a spot very similar to the one in Fig. 1(a). However, if the particle positions are correlated, the final position of the electron would depend on the final position of the ion. In this case, the image obtained after filtering the coincident electrons would be modulated since the detected ions pass through specific regions of the mask, here the CNRS letters. This spatial modulation of the coincident electrons depends on the correlation quality. The ghost imaging directly reproduces the mask only if the final $\left(X_{i}, Y_{i}\right)$ ion position and the final $\left(X_{e}, Y_{e}\right)$ electron position are proportional, which means perfectly thin and linear correlation curves in $X$ and $Y$ coordinates $\left(X_{i}\right.$ versus $X_{e}$ and $Y_{i}$ versus $\left.Y_{e}\right)$. This is nearly the case in our system that presents, indeed, a very thin and linear correlation. The thinness of the correlations is not limited by the resolution of the electron TPSD, but by the ionization conditions. The optimization of these conditions (Doppler selection, ionization at threshold, proper spatial section of the ionization region, etc.) is discussed in detail in Ref. [12]. The result is that our system has a quite good correlation quality and this allowed us to get the clear images of Fig. 1(c). 


\section{ELECTRON GHOST IMAGING}

We now present the configuration called electron ghost imaging (eGI), where all the voltages are set in a way that the ions are accelerated toward the TPSD and the electrons are accelerated toward the same mask as used before and the TSD, as shown in the scheme of Fig. 2. In this case, the electrons (in blue) will only be detected if they pass through the letters of the mask. On the other side, the extracted ions (in red) fly through the opposite part of the spectrometer and are all detected by the TPSD. Figure 2(a) shows the raw image of all the ions detected by the TPSD at the end of an acquisition sequence. If we now display only the ions that are each in coincidence with an electron using the TOF signals delivered by the TSD and the TPSD, we obtain the image displayed in Fig. 2(c). Here we clearly see on this selected TPSD image a ghost image of the mask placed in front of the TSD. As explained previously in the iGI configuration, this imaging process is based on the coincidence criterion and the position correlation quality. For the coincidence criterion, as seen in Fig. 2(d), the electron-ion relative TOF in this eGI configuration is about $8-9 \mu \mathrm{s}$. This value is slightly different from the iGI case because our time-of-flight spectrometer is not symmetric.

\section{DISCUSSION}

The ion ghost image [Fig. 1(c)] and the electron one [Fig. 2(c)] correspond to almost not zoomed and perfect images of the mask [Figs. 1(b) and 2(b)]. This is fortunate and occurs only because our spectrometer is almost symmetric. However, we stress that in other conditions, the zoom capability could be a very powerful tool to enhance spatial resolution. It can be used to see structures that would have been below the spatial resolution of a system. For instance, numerous ion and electron setups (time-of-flight mass spectrometry, electron microscopy, velocity map imaging, etc.), which use MCPs as a spatial resolution detector, with the resolution being typically $\sim 50 \mu \mathrm{m}$ (for segmented anodes, delay lines, or phosphor screen), could be enhanced by using zoomed ghost imaging $[13,14]$. Furthermore, providing that the correlation between ion and electron positions stays in a one-to-one correspondence, a dynamical or postmathematical treatment of the image can be used to correct the ghost image, even if some aberrations appear [12].

\section{CONCLUSION}

We have performed ghost imaging using a source of correlated ions and electrons with high visibility. Thanks to linear and thin $X$ and $Y$ correlation curves, the ghost images directly represent the physical mask. In more complex cases of nonlinear and ideal correlation, for instance due to modifications occurring on one trajectory after a zoom or a focusing process, posttreatment of the image is needed to more precisely reproduce the physical mask.

It is worth mentioning that the use of the time information from the TPSD and the TSD was useful here only to ensure the coincidence between the electron and the ion that also helps to reduce background noise. Note that the false coincidence rate is, in this set of experiments, less than $0.1 \%$. However, we would like to stress that the exact same method can be used even without accurate time resolution. For instance, in our experiment, we have 100 ps time resolution but, due to the low repetition rate $(\simeq 1 \mathrm{kHz})$, we could have used detectors with just a few-hundreds-of-microsecond time resolution, since the only mandatory condition is to be able to identify a coincident ion and electron pair. A simple position-sensitive device (PSD), on one side, and a single-particle detection capability, on the mask side, is enough to perform the ghost imaging. The only requirement is that we can, with low ambiguity, correlate the arrival of the particle on the mask side with the correlated particle (that is originating from the same ionization event) on the PSD side. For example, to test this hypothesis, we have increased the time coincidence window from the $200 \mathrm{~ns}$ value to a value of $20 \mu \mathrm{s}$, and the false coincidence rate only rises to a value of $5 \%$ and the ghost image of the mask, although slightly blurry, is still clearly visible. Therefore, our ghost-imaging method can be used in any system presenting a target, for instance using a detector of secondary electrons produced by the particles hitting the sample with only a crude time resolution sufficient enough to allow coincident ion and electron pair identification with a good signal-to-noise ratio.

Our ghost imaging can be more useful for surface science and nanophysics in a general way using the second particle to get information that the first one does not provide, for example, using energy or time information provided by the other particle (using adequate detectors); for instance, if the standard analyzer is an energy analyzer without any spatial resolution such as in the electron energy-loss spectroscopy [15] method, or inversely if the standard analyzer is a positionsensitive analyzer without any energy resolution such as in a conventional microscopic transmission electron microscope (TEM) [16], scanning electron microscope (SEM) [17], or scanning transmission electron or ion microscope (STEM [18], STIM [19,20]). The method is very general and the information can also be given using not only spatial but, for instance, time or energy resolution. Furthermore, because the method works for both ions and electrons, it can combine the advantage of both species. As a single example, an electron beam usually has a better spatial resolution that an ion one, whereas an ion beam has more chemical and impact properties than electrons. This ion-electron ghost-imaging techniques can also be very beneficial for the imagery systems based on electrons or ions since they enable one to improve the resolution of the direct imaging systems and to reduce the image-acquisition time or to reduce the sample damage by reducing the amount of the probe particles, for instance, when combined with arbitrarily shaped electron/ion bunches using a laser shaped by spatial light modulators [21].

These primary results are promising and can also open the door for applications especially in quantum physics and fundamental tests such as tests of Einstein-Podolsky-Rosen (EPR) entanglement, Bell's inequalities, or to improve ion and electron interferometry [22].

\section{ACKNOWLEDGMENTS}

This work was supported by the Fond Unique Interministériel (Grant No. IAPP-FUI-22) COLDFIB, the ANR/DFG HREELM, and CEFIPRA Grant No. 5404-1. 
[1] D. V. Strekalov, A. V. Sergienko, D. N. Klyshko, and Y. H. Shih, Observation of Two-Photon "Ghost" Interference and Diffraction, Phys. Rev. Lett. 74, 3600 (1995).

[2] T. B. Pittman, Y. H. Shih, D. V. Strekalov, and A. V. Sergienko, Optical imaging by means of two-photon quantum entanglement, Phys. Rev. A 52, R3429 (1995).

[3] R. S. Bennink, S. J. Bentley, and R. W. Boyd, "Two-Photon" Coincidence Imaging with a Classical Source, Phys. Rev. Lett. 89, 113601 (2002).

[4] A. Gatti, E. Brambilla, M. Bache, and L. A. Lugiato, Ghost Imaging with Thermal Light: Comparing Entanglement and ClassicalCorrelation, Phys. Rev. Lett. 93, 093602 (2004).

[5] R. I. Khakimov et al., Ghost imaging with atoms, Nature (London) 540, 100 (2016).

[6] S. Li, F. Cropp, K. Kabra, T.J. Lane, G. Wetzstein, P. Musumeci, and D. Ratner, Electron Ghost Imaging, Phys. Rev. Lett. 121, 114801 (2018).

[7] J. C. H. Spence, Outrunning damage: Electrons vs Xrays-timescales and mechanisms, Struct. Dynam. 4, 044027 (2017).

[8] O. Katz, Y. Bromberg, and Y. Silberberg, Compressive ghost imaging, Appl. Phys. Lett. 95, 131110 (2009).

[9] P. A. Morris, R. S. Aspden, J. E. C. Bell, R. W. Boyd, and M. J. Padgett, Imaging with a small number of photons, Nat. Commun. 6, 5913 (2015).

[10] M. J. Padgett and R. W. Boyd, An introduction to ghost imaging: quantum and classical, Philos. Trans. R. Soc., A 375, 20160233 (2017).

[11] P. W. Hawkes and J. C. Spence, Springer Handbook of Microscopy (Springer Nature, New York, 2019).
[12] C. Lopez, A. Trimeche, D. Comparat, and Y. J. Picard, RealTime Trajectory Control of Deterministically Produced Ions, Phys. Rev. Appl. 11, 064049 (2019).

[13] J. H. Jungmann and R. M. Heeren, Detection systems for mass spectrometry imaging: A perspective on novel developments with a focus on active pixel detectors, Rapid Commun. Mass Spectrom. 27, 1 (2013).

[14] L. M. Hirvonen and K. Suhling, Wide-field TCSPC: methods and applications, Meas. Sci. Technol. 28, 012003 (2017).

[15] R. F. Egerton, Electron energy-loss spectroscopy in the TEM, Rep. Prog. Phys. 72, 016502 (2008).

[16] H. Kohl and L. Reimer, Transmission electron microscopy physics of image formation and microanalysis, Vol. 36 (Springer, New York, 2013).

[17] J. I. Goldstein et al., Scanning Electron Microscopy and X-ray Microanalysis (Springer, New York, 2017).

[18] S. J. Pennycook and P. D. Nellist, Scanning Transmission Electron Microscopy: Imaging and Analysis (Springer Science \& Business Media, New York, 2011).

[19] J. Overley, R. Schofield, J. MacDonald, and H. Lefevre, Energy-loss image formation in scanning transmission ion microscopy, Nucl. Instrum. Methods Phys. Res., Sect. B 30, 337 (1988).

[20] M. B. H. Breese, J. P. Landsberg, P. J. C. King, G. W. Grime, and F. Watt, Applications of scanning transmission ion microscopy, Nucl. Instrum. Methods Phys. Res., Sect. B 64, 505 (1992).

[21] A. McCulloch et al., Arbitrarily shaped high-coherence electron bunches from cold atoms, Nat. Phys. 7, 785 (2011).

[22] F. Hasselbach, Progress in electron- and ion-interferometry, Rep. Prog. Phys. 73, 016101 (2009). 